\title{
Thermal condensation in a turbulent atomic hydrogen flow ${ }^{\star}$
}

\author{
E. Audit ${ }^{1}$ and P. Hennebelle ${ }^{2}$ \\ 1 Service d'Astrophysique, CEA/DSM/DAPNIA/SAp, C. E. Saclay, 91191 Gif-sur-Yvette Cedex, France \\ e-mail: edouard.audit@cea.fr \\ 2 Laboratoire de radioastronomie millimétrique, UMR 8112 du CNRS, École normale supérieure et Observatoire de Paris, \\ 24 rue Lhomond, 75231 Paris Cedex 05, France \\ e-mail: patrick.hennebelle@ens.fr
}

Received 15 June 2004 / Accepted 30 October 2004

\begin{abstract}
We present a numerical and analytical study of the thermal fragmentation of a turbulent flow of interstellar hydrogen. We first present the different dynamical processes and the large range of spatial (and temporal) scales that need to be adequately represented in numerical simulations. Next, we present bidimensional simulations of turbulent converging flows which induce the dynamical condensation of the warm neutral phase into the cold phase. We then analyse the cold structures and the fraction of unstable gas in each simulation, paying particular attention to the influence of the degree of turbulence. When the flow is very turbulent a large fraction of the gas remains in the thermally unstable domain. This unstable gas forms a filamentary network. We show that the fraction of thermally unstable gas is strongly correlated with the level of turbulence of the flow. We then develop a semi-analytical model to explain the origin of this unstable gas. This simple model is able to quantitatively reproduce the fraction of unstable gas observed in the simulations and its correlation with turbulence. Finally, we stress the fact that even when the flow is very turbulent and in spite of the fact that a large fraction of the gas is maintained dynamically in the thermally unstable domain, the classical picture of a 2-phase medium with stiff thermal fronts and local pressure equilibrium turns out to be still relevant in the vicinity of the cold structures.
\end{abstract}

Key words. hydrodynamics - instabilities - ISM: kinematics and dynamics - ISM: structure - ISM: clouds

\section{Introduction}

The dynamics of the interstellar gas is of great importance to understand the formation of structures such as molecular clouds and it is therefore determinant in the star formation process.

In this respect, the neutral atomic hydrogen (HI) is crucial since it represents more than $50 \%$ of the ISM and since the molecular clouds form through the condensation of $\mathrm{HI}$ gas. Previous numerical models attempting to simulate the ISM at a scale of about $1 \mathrm{kpc}$ and to form molecular clouds selfconsistently have not considered heating and cooling functions that allow thermal bistability between 100 and $8000 \mathrm{~K}$ (e.g Passot et al. 1995; Vázquez-Semadeni et al. 1996; Korpi et al. 1999; Ballesteros-Paredes et al. 1999) and have a numerical resolution which is not appropriate to adequately describe this physics down to the scale of the CNM structures. Therefore it is currently unclear and indeed almost unexplored to what extent the physics of HI may or may not have a significant influence on the formation and the evolution of molecular clouds.

From the theoretical point of view (Field et al. 1969; McKee \& Ostriker 1977; Wolfire et al. 1995) as well as from

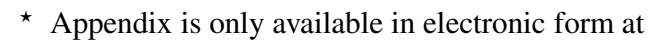
http://www. edpsciences.org the observational one (Low et al. 1984; Boulanger \& Pérault 1988; Kulkarni \& Heiles 1987; Troland \& Heiles 2003), it is now well established that $\mathrm{HI}$ is a thermally bistable medium which at thermal equilibrium and for a thermal pressure close to about (in the vicinity of the sun) $4000 \mathrm{~K} \mathrm{~cm}^{-3}$, can be in two different thermodynamical states, namely a warm and diffuse phase (WNM) and a cold and dense one (CNM) roughly in pressure equilibrium.

The linear stability analysis (Field 1965), the quasi-static thermal front propagation (Zel'dovich \& Pikel'ner 1969; Penston \& Brown 1970) and more generally the non-linear development of a single structure (see Meerson 1996, for a review; and Sánchez-Salcedo et al. 2002 for a recent study) have been under investigation for a long time and are reasonably well understood. However, it is only recently that the behaviour of a thermally bistable flow in the fully dynamical or turbulent regime has been investigated.

\subsection{Previous work}

In the context of the solar chromosphere and corona, Dahlburg et al. (1987) and Karpen et al. (1988) performed 2D simulations of gas that may undergo thermal instability. They considered random velocity perturbations in an initially thermally 
unstable gas and study the development of cold structures. They note that large amplitude velocity perturbations can prevent the formation of condensed structures.

Kovalenko \& Shchekinov (1999) and Hennebelle \& Pérault (1999) investigated the possibility that a converging flow may dynamically trigger the thermal transition from the WNM (thermally stable) phase into the CNM phase. They performed 1D simulations and show that if the perturbation lasts long enough (more than a cooling time) and is strong enough (velocity must be comparable to the sound speed), the thermal transition is possible, i.e. part of the WNM phase condenses into CNM leaving a cold structure embedded in a warm surrounding medium. Their underlying idea is that an external forcing like bubble expansion or any phenomena generating systematic or turbulent motions may promote the formation of cold structures. A similar picture has been investigated by Koyama \& Inutsuka (2000) who simulate a shock propagating in HI and include $\mathrm{H}_{2}$ formation. Hennebelle \& Pérault (2000) have also considered the case of a magnetic field, important in the context of the ISM, which is initially oblique with respect to the velocity field. They show that whatever the value of the magnetic intensity, thermal condensation is still possible provided the initial angle between $\boldsymbol{B}$ and $\boldsymbol{V}$ is small enough (the smallest angle below which thermal condensation is always possible being about $20^{\circ}$ ) and conclude that in the interstellar atomic hydrogen no correlation between the magnetic intensity and the density is expected (except locally in the vicinity of a strong shock).

This paradigm has been further investigated by Koyama \& Inutsuka (2002) who simulate in 2D a shock propagating in HI. They show that several structures of CNM form close to the shock interface and find that the velocity dispersion of the cold structures is about $5-6 \mathrm{~km} \mathrm{~s}^{-1}$, i.e. a fraction of the sound speed of the WNM.

Gazol et al. (2001) performed 2D simulations of HI at a scale of about $1 \mathrm{kpc}$ and a numerical resolution of about $5 \mathrm{pc}$ with a turbulent forcing that mimics star formation. They were the first to report an important fraction $(\simeq 50 \%)$ of thermally unstable gas in their simulation. They confirm this result by performing numerical simulations with the same forcing and a numerical resolution of about 0.1 pc (Vázquez-Semadeni et al. 2003).

Kritsuk \& Norman (2002a,b) performed 3D simulations of $\mathrm{HI}$ with a thermal forcing that mimics the random fluctuations of the heating rate derived by Parravano et al. (2002). Their aim is to show that interstellar turbulence may be generated by thermal instability.

Finally, Piontek \& Ostriker (2004) performed 2D simulations to study the development of the magnetorotational instability as well as the thermal instability in the magnetised warm atomic interstellar gas.

\subsection{Outline of the paper}

In this paper we further investigate the dynamical properties of HI gas by the mean of 2D numerical simulations. We explore the behaviour of $\mathrm{HI}$ focusing on the formation of cold structures during the collision between two streams (converging flow) of WNM. We pay special attention to the structure morphology, internal velocity dispersion and to the fraction of gas in the cold and warm phase and in an intermediate thermally unstable state. Our aim is to understand how those features depend on the turbulence of the flow.

In the second section of the paper we present the equations, describe the thermal processes, the numerical scheme and the initial and boundary conditions. We also discuss the drastic numerical resolution needed in order to properly describe the HI flow. In the third section we present our numerical results for a large-scale flow that is weakly or very turbulent and present a statistical analysis of the simulations. In the fourth section we emphasize the correlation between turbulence and the fraction of thermally unstable gas. We then develop a semi-analytical model to understand the physical mechanisms responsible for this phenomenon. The fifth section summarises our results and concludes the paper.

\section{Equations, numerical requirements and initial conditions}

\subsection{Equations and notations}

In this paper we consider the Euler equations for an optically thin gas. The gas is able to cool radiatively and is heated by an external radiation field. The equations governing the evolution of the fluid are the classical equations of hydrodynamics, where a cooling function is added in the energy conservation equation:

$\partial_{t} \rho+\nabla \cdot[\rho u]=0$,

$\partial_{t} \rho u+\nabla \cdot[\rho u \otimes u+P]=0$,

$\partial_{t} E+\nabla \cdot[u(E+P)]=-\mathcal{L}(\rho, T)$,

where $\rho$ is the mass density, $u$ the velocity, $P$ the pressure, $E$ the total energy and $\mathcal{L}$ the cooling function (see next section). The gas is assumed to be a perfect gas with $\gamma=5 / 3$ and with a mean molecular weight $\mu=1.4 m_{\mathrm{H}}$, where $m_{\mathrm{H}}$ is the mass of the proton.

The above equations are solved using a second-order Godunov method for the conservative part. The cooling is applied after the hydrodynamical step using an implicit scheme and subcycling when the cooling time is much smaller than the time step.

\subsection{Thermal processes}

To simulate $\mathrm{HI}$ it is important to adopt a model for thermal processes which on one hand is realistic enough and on the other hand not too computationally expensive. We have followed closely the work of Wolfire et al. $(1995,2003)$, trying to include only the most relevant processes to our study. The UV flux is taken equal to $G_{0} / 1.7$, where $G_{0}$ is the Draine's flux (Draine 1978).

We have only kept the following cooling processes, which are dominant in the physical conditions of our simulations:

- cooling by fine-structure lines of CII (92 K); 
- cooling by fine-structure lines of OI (228 and $326 \mathrm{~K})$;

- cooling by $\mathrm{H}$ (Ly $\alpha$ line);

- cooling by electron recombination onto positively charged grains.

The heating process is the photo-electric effect on small grains and polyaromatic hydrocarbons due to the far-ultraviolet galactic radiation. We have not taken the heating due to cosmic rays and to the soft X-rays into account since these contributions appear to be small. To calculate the ionisation we use the approximation proposed by Wolfire et al. (2003).

We have compared each term with the results given in Wolfire et al. (95) as well as the thermal equilibrium curve. Very similar results have been obtained.

\subsection{Resolution requirement}

The thermal condensation of HI gas involves 4 different spatial scales, each related to a different physical mechanism that we describe below.

\subsubsection{First scale: Cooling length in the WNM}

The cooling length of the WNM, $\lambda_{\text {cool }}$ is defined as the product of the cooling time, $\tau_{\text {cool }}=C_{v} T /(\mathcal{L} \rho)$ and the sound speed, $C_{\mathrm{s}}$. It represents the scale at which the WNM is non-linearly unstable (Hennebelle \& Pérault 1999), i.e. velocity fluctuations of spatial extension $\simeq \lambda_{\text {cool }}$ and amplitude $\simeq C_{\mathrm{s}}$, can undergo a thermal contraction from WNM phase into CNM phase. For typical WNM parameters, $n_{\text {wnm }} \simeq 0.5 \mathrm{~cm}^{-3}, T_{\text {wnm }} \simeq 8000 \mathrm{~K}, \lambda_{\text {cool }}$ is about $10-20 \mathrm{pc}$ whereas for a higher density of $n_{\mathrm{wnm}} \simeq 3 \mathrm{~cm}^{-3}$, reached for example in shock or dynamically compressed layer $\lambda_{\text {cool }}$ is smaller and about $1-3 \mathrm{pc}$.

\subsubsection{Second scale: Fragment size}

The second important scale of the problem is the typical size of the fragments of CNM. It is given by the cooling length divided by the density ratio between the CNM and the WNM, $\zeta \simeq 100$. This is due to the fact that a piece of initially warm gas contracts until it reaches thermal equilibrium. Therefore $\lambda_{\text {struct }}=\lambda_{\text {cool }} / \zeta \simeq 0.1 \mathrm{pc}$. We stress that resolving this scale is essential to properly describe the structure of the cold gas. Note that $\lambda_{\text {struct }}$ is the typical size of the structures in the direction along which the gas has condensed. In the other directions, if the collapse is anisotropic, the scale is a priori different and is rather related to the initial scale of the WNM fluctuation that condensed into the CNM structure.

\subsubsection{Third scale: Field's length}

The third scale is the Field length (Field 1965) or conduction length, which is also the typical size of the front between the 2 phases. It is given by $\lambda_{\text {Field }}=\sqrt{(\kappa(T) T) /|\mathcal{L}|}$. In the WNM, the Field's length is about $10^{-1} \mathrm{pc}$ and is about $10^{-3} \mathrm{pc}$ in the CNM. Since the front propagation velocity is proportional to $\lambda_{\text {Field }}$ (Zel'dovitch \& Pikel'ner 1969; Penston \& Brown 1970), the consequences of not properly resolving this scale would be first to overestimate the propagation of the thermal front and second to overestimate the fraction of thermally unstable gas located in the front. We thus conclude that in order to properly simulate the HI gas it is essential that the effective Field length (close to the numerical resolution) in the simulation is small compared to $\lambda_{\text {struct }}$. This ensures that the growth of structures due to heat diffusion remains small compared to the growth of structures due to dynamical processes and that the fraction of thermally unstable gas stabilised by heat diffusion and located in thermal fronts is small compared to the fraction of cold gas. The importance of resolving the Field length has been carefully analysed by Koyama \& Inutsuka (2004).

\subsubsection{Fourth scale: Shocked CNM}

The fourth scale is a consequence of dynamical processes. The internal sound speed of the CNM is about 10 times smaller than the sound speed of the WNM. Consequently, it is expected that HI structures experience supersonic motions with a typical Mach number, $M$, equal to about 10 . This may occur through the collision between 2 fragments of CNM or when a fragment of CNM is swept up by a shock wave. Assuming that the polytropic index of the CNM is about 1 (i.e. CNM is isothermal), this leads to a density increase of $\rho_{\text {shock }}=M^{2} \rho_{\text {cnm }}$. The typical size of the shocked layer is thus $\lambda_{\text {shock }}=\lambda_{\text {struct }} / M^{2}$ which is about $\simeq 10^{-3}$ pc for $M=10$. If the numerical resolution is larger than $\lambda_{\text {shock}}$, the largest density reached in the simulation is underestimated and the size of the shocked layer is artificially broadened.

As we have seen, simulating the thermal condensation in HI satisfactorily would require us to treat spatial scales ranging from few 10 pc to about $\simeq 10^{-3}$ pc simultaneously.

\subsection{Boundaries and initial conditions}

Due to the large range of spatial scales involved in the interstellar medium it is not possible to properly resolve the scales associated with the microphysics (see previous section) and at the same time to treat the large-scale flow of the WNM. It is therefore necessary to make compromises between the different scales.

Since we are interested in the formation of small dense structures, we have chosen to resolve the small scales and to use a simple prescription to model the large-scale velocity field of the WNM. Therefore, the simulations used in this paper are $2 \mathrm{D}$ simulations on a $1000^{2}$ grid. In order to solve the cooling length, the size of the box is $L=20$ pc leading to a numerical resolution of $0.02 \mathrm{pc}$. This numerical resolution ensures that $\lambda_{\text {struct }}$ is well resolved, and that it is larger than the effective Field's length. However with this resolution a shocked structure is not well described for Mach numbers larger than about 3 . This means that the highest densities reached during supersonic collisions are underestimated in our simulations.

The size of the simulation box, $20 \mathrm{pc}$, is marginal in the sense that it is not enough to describe the evolution of a largescale structure of WNM. The large-scale flow is then imposed by choosing boundary conditions that mimic a converging flow at large-scale. The upper and lower sides of the simulations 


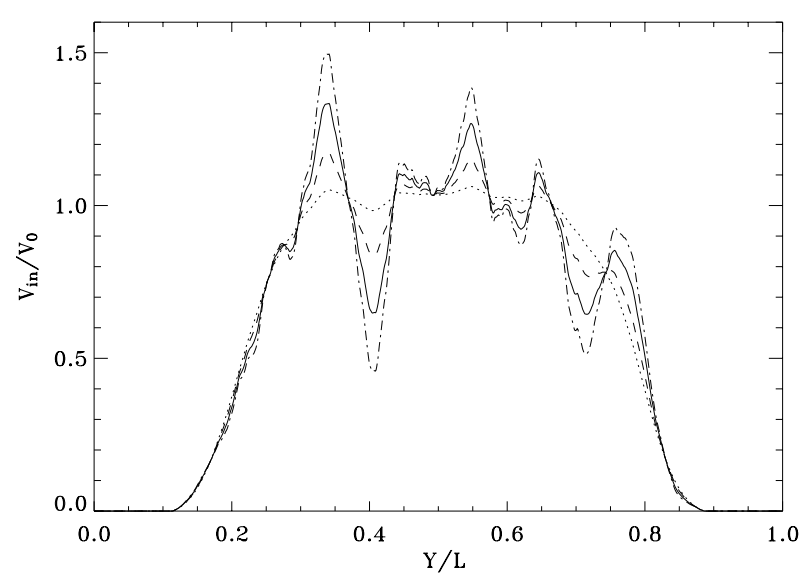

Fig. 1. Inflowing velocity field for $\epsilon=0.5$ (dotted line), $\epsilon=2$ (dashed line), $\epsilon=4$ (solid line) and $\epsilon=6$ (dot-dashed line). As can be seen, we have kept the same phase for the 3 curves.

have free boundary conditions while on the left (resp. right) side the gas is injected with a velocity $V_{\text {in }}(y)\left(\right.$ resp. $\left.-V_{\text {in }}(y)\right)$. The density and the pressure of the inflowing gas are chosen such that the gas is at thermal equilibrium in the branch of the WNM phase. It is thus thermally stable when it penetrates the simulation box.

The function $V_{\text {in }}$ describes the velocity of the inflowing gas and is given by:

$V_{\text {in }}(y)=V_{0} \quad \mathrm{e}^{-((y-L / 2) / 0.6 L)^{6}} \quad(1+U(y))$,

where $U(y)=\epsilon \sum c_{i} \cos \left(k_{i} y+\alpha_{i}\right)$.

This velocity field represents a stream of gas with a transverse spatial extension of about $L / 2$ and an average velocity equal to $V_{0}$. In order to study the influence of turbulence, this field can be modulated by the function $U(y) . \epsilon$ is the amplitude of this modulation; $k_{i}=2 \pi / \lambda_{i}$ is the wave number and the wave-length $\lambda_{i}$ lies between 2 cells and $\mathrm{L} / 4$. We have chosen a power law spectrum of index -1 for the modulation. Therefore the $c_{i}$ are defined by $c_{i} \propto k_{i}^{-1}$ and $\sum c_{i}=1$. The $\alpha_{i}$ are random phases which lie between 0 and $2 \pi$. If $\epsilon$ is small then the flow remains essentially laminar whereas it becomes turbulent if $\epsilon$ is greater than 1. Figure 1 illustrates the shape of the inflowing velocity field for different values of $\epsilon(\epsilon=0.5,2,4$ and 6).

The gas injected into the simulation box is at thermal equilibrium in the WNM branch and has a constant pressure equal to $P=7 \times 10^{-13} \mathrm{erg} \mathrm{cm}^{-3}$. The gas temperature and density are respectively $\simeq 7100 \mathrm{~K}$ and $n=0.76 \mathrm{~cm}^{-3}$.

We have compared $1 \mathrm{D}$ results involving a thermal transition between the 2 phases with the code used by Hennebelle \& Pérault (1999) and obtained very similar results within a few percent accuracy even though the code used in this work does not include viscosity and thermal conduction.

\section{Results}

In this section we qualitatively describe results for a weakly turbulent $(\epsilon=0.5)$ and for a very turbulent forcing $(\epsilon=4)$. We then present a statistical analysis for the 4 cases, $\epsilon=0.5,2,4$ and 6 considering the gas fraction in the different phases and the properties of the CNM structures formed in the simulations.
Initially, there is only warm gas in the simulation box. Then the two facing incoming flows generate a region of higher density and pressure in the center. This region is thermally unstable and cold structures start to form. After some time (from 5 to $15 \mathrm{Myr}$ ), the simulation reaches a permanent regime, i.e. the mass fraction in the different phases, the statistical properties of the cold structures, their abundance etc, remain constant. All the results presented below are given for this permanent regime.

\subsection{Weakly turbulent flow}

When the flow is weakly turbulent, the ram pressure of the 2 incoming flows induces the formation of a high pressure central layer which is bounded by 2 accretion shocks. This high pressure gas has a density of about $3 \mathrm{~cm}^{-3}$ and a temperature of about $10^{4} \mathrm{~K}$. Due to the high pressure in the central region a roughly homologous expanding velocity field develops in the transverse direction and part of the gas escapes the box continuously. The velocity field as well as the density field in the central region can be seen in Fig. 2 which displays a zoom of $4 \times 4 \mathrm{pc}^{2}$ around the box centre (located at $x=10 \mathrm{pc}$, $y=10 \mathrm{pc}$ ). The layer is out of thermal equilibrium and its central part becomes thermally unstable. Part of the warm gas condenses into cold gas. This behaviour is indeed very similar to the 1D situation studied by Hennebelle \& Pérault (1999). However in the present case because of the weak turbulence, the cold gas layer is unstable and fragments in several parts as can be seen in Fig. 2 where about 10 fragments have formed. Their size ranges from about 0.05 to $0.3 \mathrm{pc}$. Very sharp thermal fronts (2-3 pixels) bound the structures and connect them to the warm surrounding medium. Due to the high pressure induced by the incoming flow, the density of the structures is rather high and ranges from a few 100 to $\simeq 2000 \mathrm{~cm}^{-3}$. The fragments are sometimes connected by a thin layer of low density gas.

This situation is very similar to the $2 \mathrm{D}$ numerical simulations of Koyama \& Inutsuka (2002) who study the thermal transition induced by a shock propagating in the warm phase. As in our case, Koyama \& Inutsuka (2002) find that the shocked layer fragments in few cold clouds of about $0.1 \mathrm{pc}$.

Figure 3 gives a more accurate description of the thermal state of the gas in the simulation. It shows the gas fraction in the pressure-density diagram. The full line is the thermal equilibrium curve. The green lines are the isothermal curves $T=5000 \mathrm{~K}$ and $T=200 \mathrm{~K}$ and the blue line is the Hugoniot curve of shocked gas corresponding to our initial conditions. Most of the warm gas is located between the Hugoniot curve and the isothermal curve $T=5000 \mathrm{~K}$ whereas most of cold gas is very close to the thermal equilibrium curve. There is almost no thermally unstable gas.

\subsection{Very turbulent flow}

Figure 4 displays the density and velocity fields in the whole box for the very turbulent forcing $(\epsilon=4)$ at time $t=18.93 \mathrm{Myr}$. 


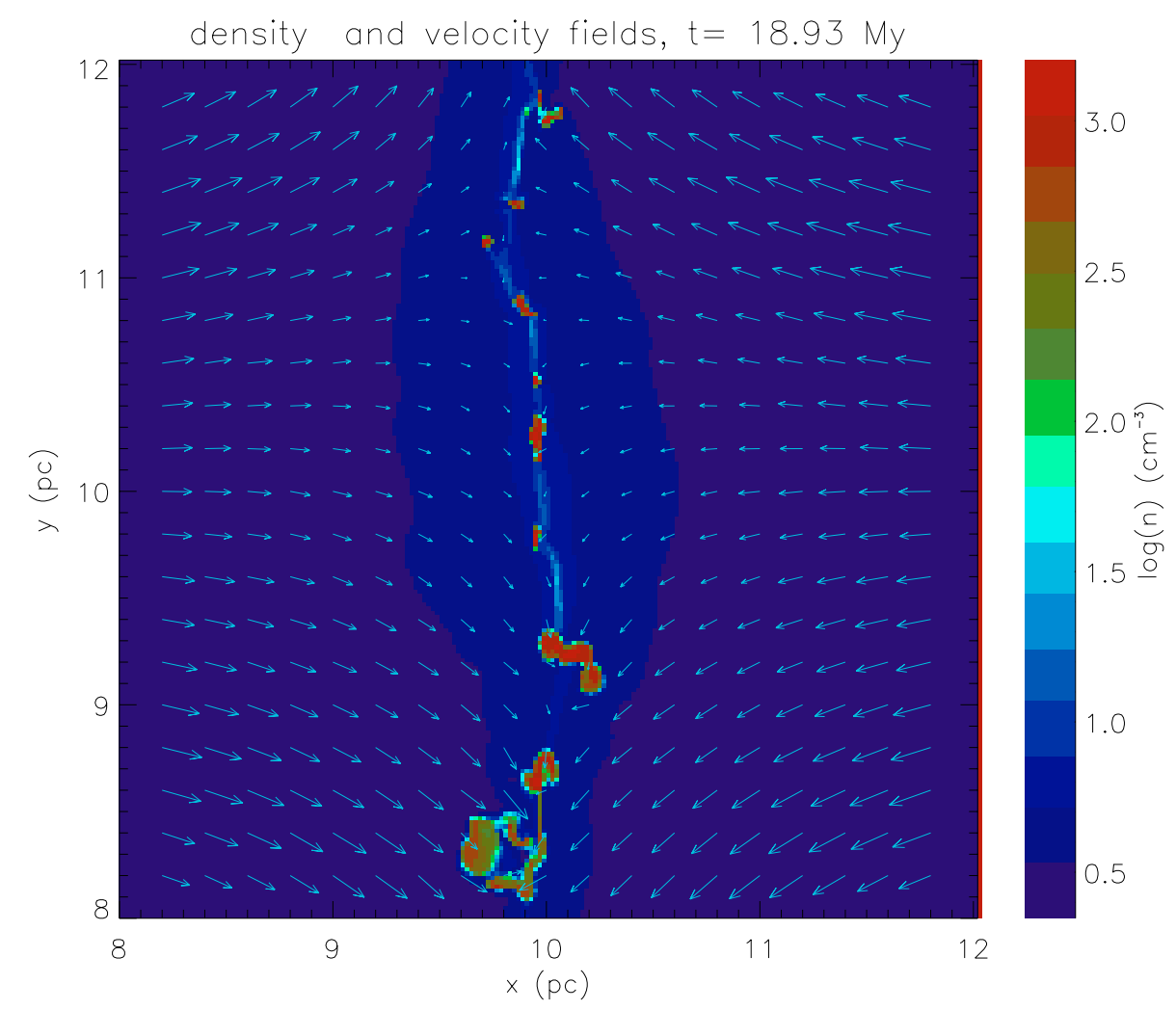

Fig. 2. Density and velocity fields in the central region $\left(4 \times 4 \mathrm{pc}^{2}\right.$ around the center located at $x=10 \mathrm{pc}$ and $\left.y=10 \mathrm{pc}\right)$ of the simulation box $\left(20 \times 20 \mathrm{pc}^{2}\right)$ for $\epsilon=0.5$ (weakly turbulent flow) at time $t=18.93$ Myr. The longest arrow represents a velocity of about $15 \mathrm{~km} \mathrm{~s}^{-1}$.

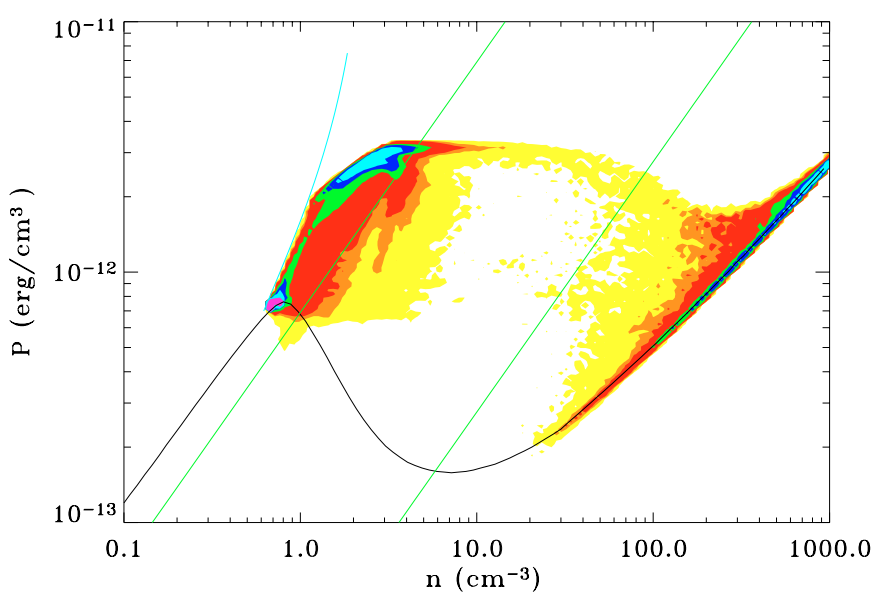

Fig. 3. Gas mass fraction in the pressure-density diagram for the $\epsilon=0.5$ simulation. The colours correspond respectively to gas mass fraction (in arbitrary units) of $(1,5,10,50,100,200,1000)$ for (yellow, orange, red, green, dark blue, light blue, pink). The full black line is the thermal equilibrium curve. The green lines are the isothermal curves $T=5000 \mathrm{~K}$ and $T=200 \mathrm{~K}$ and the blue line is the Hugoniot curve of shocked gas corresponding to our initial conditions.

The stiff shear of the gas that penetrates the box generates turbulence quickly.

The interface between the 2 flows is very irregular and significantly distorted. The density field is much more complex than in the previous case. As illustrated in Fig. 7, less material is at a density of more than few $100 \mathrm{~cm}^{-3}$ and a significant fraction of the gas is at intermediate density around $5 \mathrm{~cm}^{-3}$ and temperature smaller than $5000 \mathrm{~K}$, i.e. in a thermally unstable state. Moreover, as can be seen by comparing Figs. 3 and 7 the average thermal pressure is higher in the weakly turbulent case. This is due to the fact that the kinetic energy of the incoming flow (which is almost constant in all simulations) is converted into both thermal pressure and turbulent motions. Therefore, if the turbulent motions are high, the thermal pressure should be lower.

As can be seen in Figs. 5 and 6 which displays respectively the density and velocity fields and the temperature field and Mach number of Fig. 4 between $x=8$ and $12 \mathrm{pc}$ and $y=10.5$ and $14.5 \mathrm{pc}$, the thermally unstable gas is very filamentary and presents a complex structure (we use the word filament for the elongated structures seen in our 2D simulations. In 3D these could become either sheets or filaments, although we believe that filaments are more likely since sheets would be more easily broken by turbulent motions).

Several interesting features appear. The different phases are highly interwoven with pockets of warm gas embedded in filaments of cooler gas. This is particularly obvious in Fig. 6 around $x \simeq 11.5 \mathrm{pc}$ and $y \simeq 11.5 \mathrm{pc}$.

In spite of the presence of this unstable gas, the sharp fronts separating the cold and warm phases are still obvious as can be seen for the structure located at $x=9 \mathrm{pc}$ and $y=13.8 \mathrm{pc}$. This relatively unsurprising result means that even in a very dynamic medium the 2-phase behaviour is not suppressed and may indeed be locally rather similar to the standard equilibrium 2-phase model. 


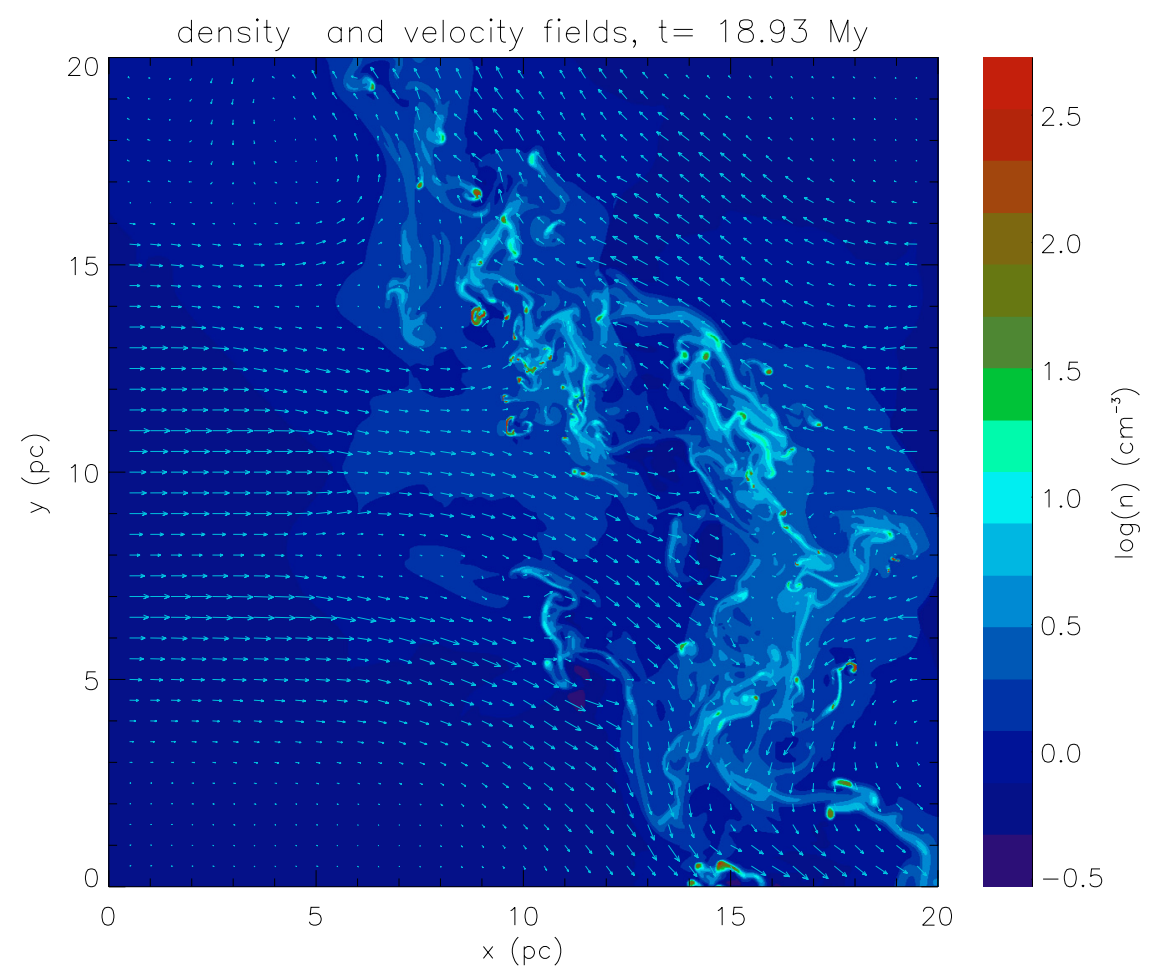

Fig. 4. Density and velocity fields for a very turbulent forcing $(\epsilon=4)$. The longest arrow represents a velocity of about $17 \mathrm{~km} \mathrm{~s}^{-1}$.

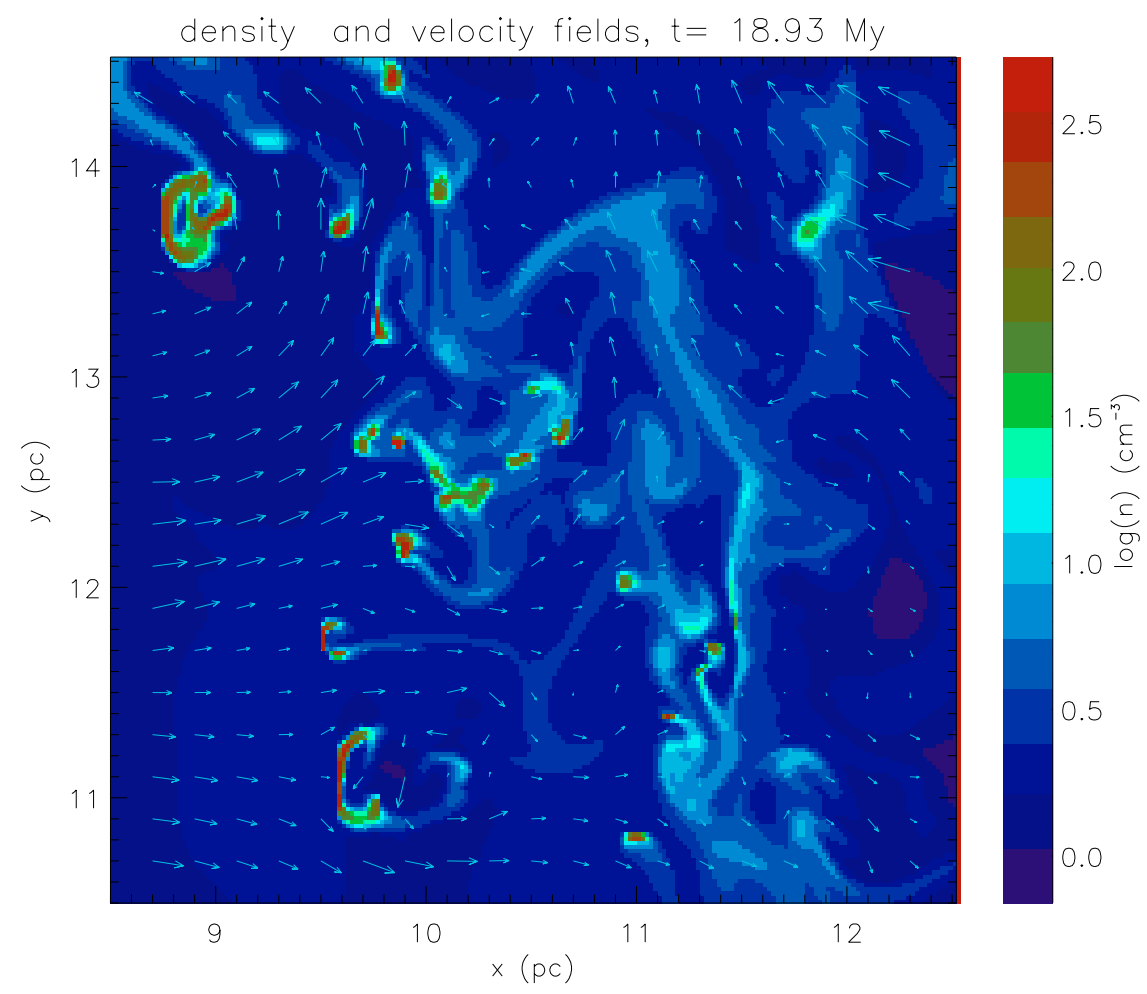

Fig. 5. Spatial zoom of Fig. 4. The longest arrow represents a velocity of about $13 \mathrm{~km} \mathrm{~s}^{-1}$.

Sharp transitions can be seen between the warm phase and the filaments of thermally unstable gas as well (see for example the front at $x \simeq 11.2, y \simeq 11.3 \mathrm{pc}$ ).

There are more cold structures than in the previous case $(\epsilon=0.5)$ but they are relatively less dense. This suggests that turbulence promotes the fragmentation of thermally unstable medium and that due to the more random motions (i.e. less organised than for the case $\epsilon=0.5$ ) the average thermal pressure (due to the high ram pressure) of the medium is reduced.

The cold structures are clearly associated with the unstable gas since they are often linked to other cold structures by a 


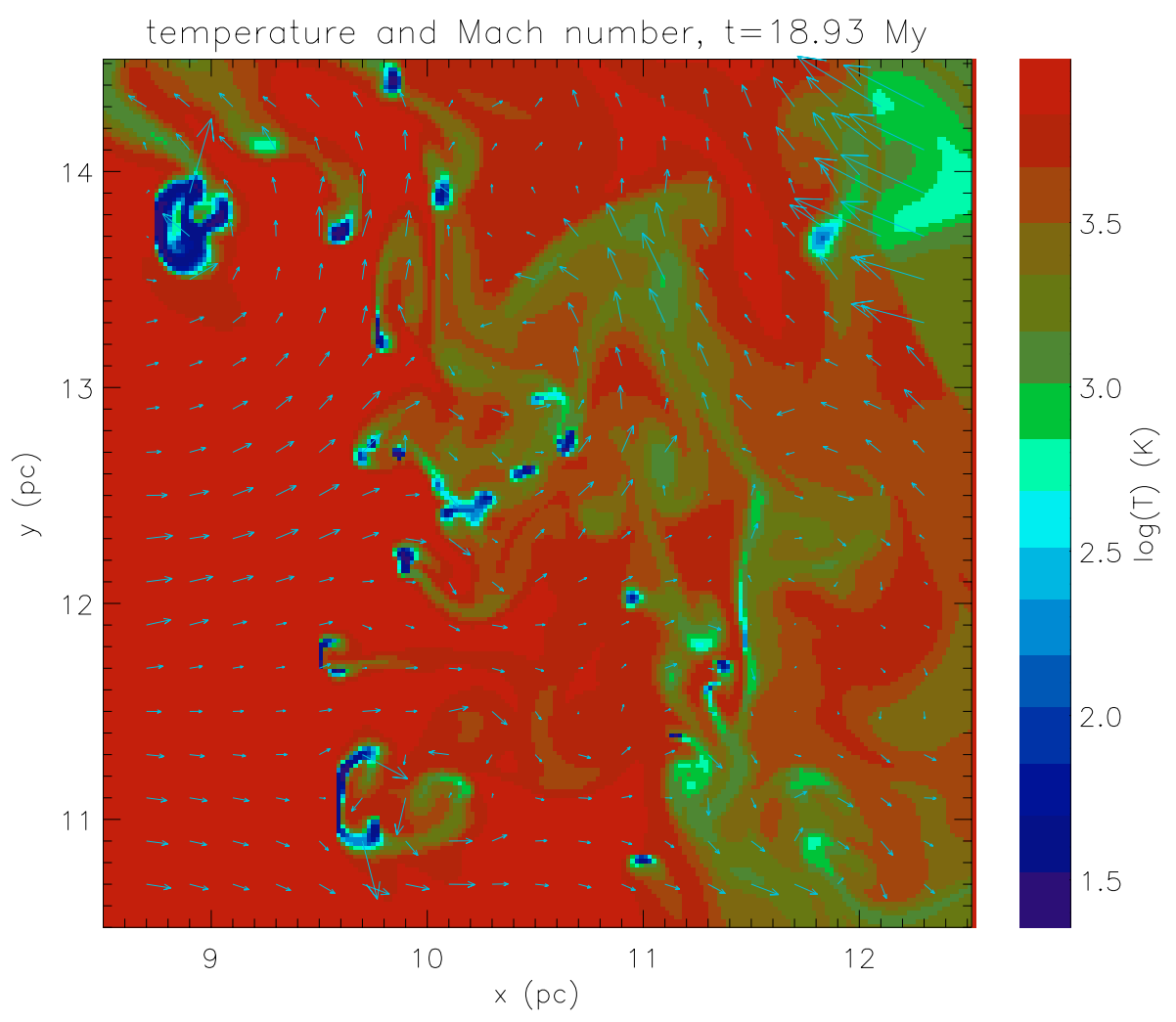

Fig. 6. Temperature and Mach number corresponding to Fig. 5. The longest arrow represents a Mach number of about 10.

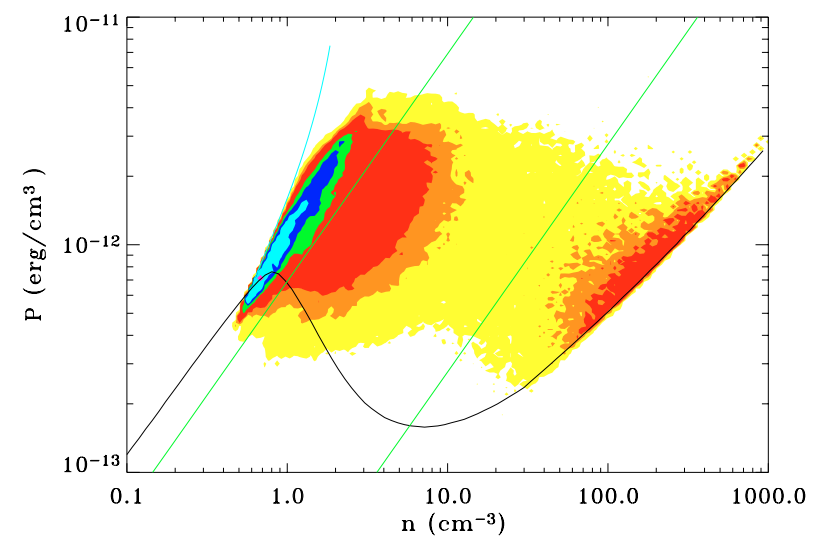

Fig. 7. Same as Fig. 3 for $\epsilon=4$.

filament of unstable gas and sometimes embedded in such a filament.

Note that the size of the smallest cold structures is close to the mesh of our simulation. This means, as pointed out in Sect. 2, that numerical convergence is clearly not reached for those small structures. Moreover as can be seen in Fig. 5, the cold fragments have a complex internal structure that is also smoothed by the lack of resolution.

We would like to stress the fact that in the dynamical regime, the 2-phase behaviour is clearly not erased and that the description of the medium as a continuum of phases would not be correct. There is thermally unstable gas but it is highly structured, very filamentary and connected to the denser thermally stable gas which is very differently structured. This means that new phenomena due to the thermal nature of the flow rather than simple disappearance of the two phase behaviour occur in this regime.

\subsection{Gas fraction in the different phases}

In this section, we analyse the temperature, density and pressure distributions obtained in the 2 cases presented previously $(\epsilon=0.5,4)$ and 2 additional cases $(\epsilon=2$ and 6$)$. For simplicity and in order to give simple trends, we call thermally unstable gas the gas having a temperature between 5000 and $200 \mathrm{~K}$, whereas the gas with a temperature below $200 \mathrm{~K}$ is defined as cold gas.

Figure 8 shows histograms of the fraction of gas $(X)$ as a function of temperature, density and pressure in the four cases $\epsilon=0.5,2,4$ and 6 for 4 time steps: $t=11.6,15.8,17.9$ and $20 \mathrm{Myr}$.

For the case $\epsilon=0.5$, the distributions at the 4 time steps presented are very similar, which means that the permanent regime is reached quickly. The fraction of thermally unstable gas is low $(\simeq 10 \%)$ and the fraction of CNM is about $30 \%$. There is almost no gas at intermediate density $\left(n \simeq 10 \mathrm{~cm}^{-3}\right)$ whereas $X$ is almost constant for a density between 100 and $1000 \mathrm{~cm}^{-3}$. It then drops stiffly for $n>1000 \mathrm{~cm}^{-3}$. The pressure fluctuates over 1 order of magnitude. The peak at $\log \left(P / P_{\text {med }}\right) \simeq-0.1$ is due to the preshocked gas whereas the peak at $\simeq 0.5$ is the postshocked gas ( $P_{\text {med }}$ is the median pressure).

In the case $\epsilon=2$, the fraction of cold gas is slightly lower (20\%) than for $\epsilon=0.5$ whereas the fraction of thermally 

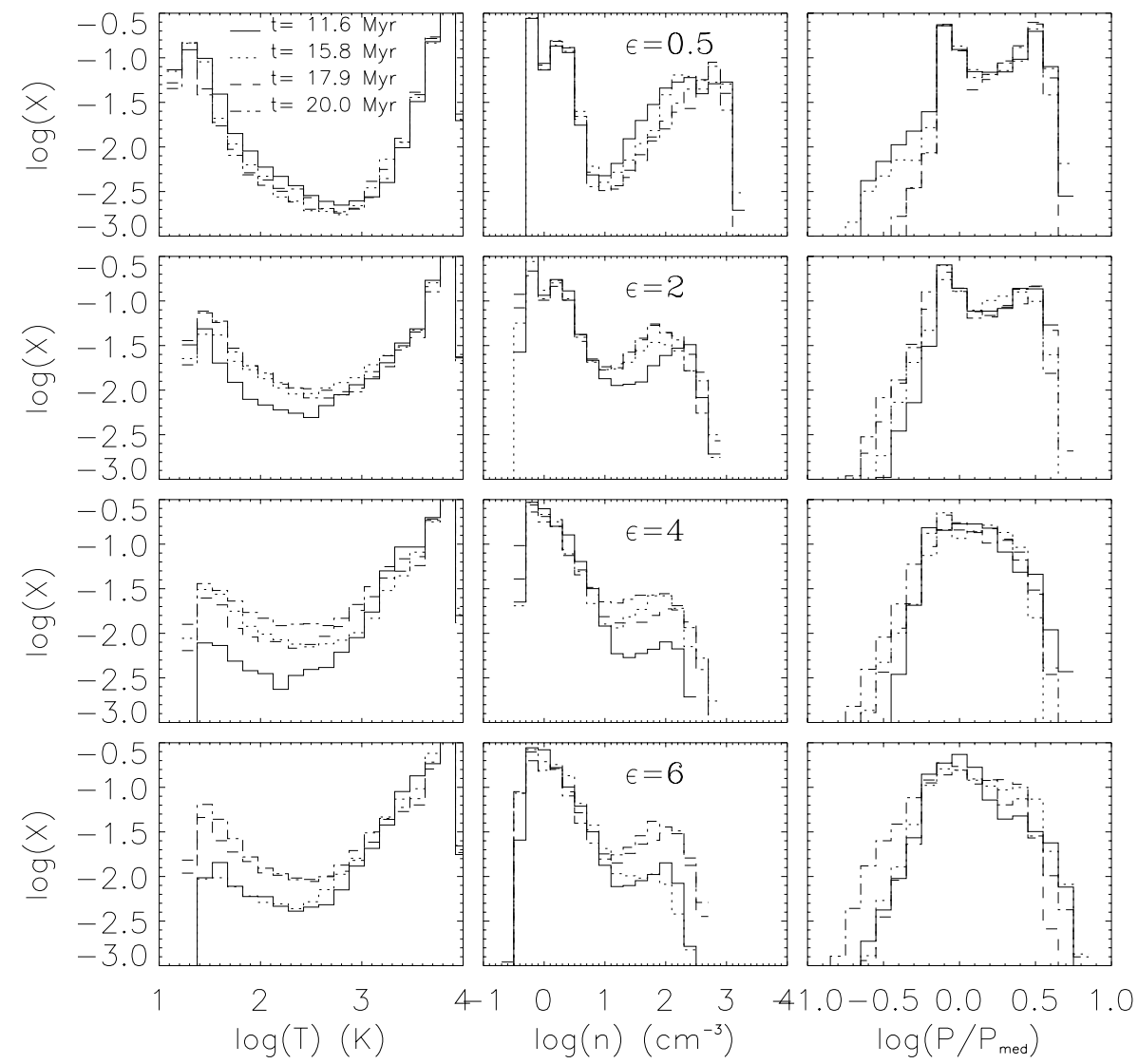

Fig. 8. Fraction of gas as a function of temperature, density and pressure for $\epsilon=0.5, \epsilon=2, \epsilon=4$ and $\epsilon=6$ at times $t=11.6,15.6$ and $20 \mathrm{Myr}$.

unstable gas is larger (20\%). The fraction of gas at a density above $10 \mathrm{~cm}^{-3}$ is lower at the first time step than later on which means that the permanent regime takes longer to reach.

For the cases $\epsilon=4$ and 6 , these trends are even clearer. It is seen that the distribution at time $t=11.6 \mathrm{Myr}$ is significantly different from the distributions at time $t=15.8,17.9$ and $20 \mathrm{Myr}$. The fraction of dense gas is smaller at $t=11.6 \mathrm{Myr}$ by approximately a factor of 3 . This means that the permanent regime takes much longer to reach than for the previous cases. Anticipating the mechanism that will be discussed in Sect. 4, we interpret this result as a consequence of the fact that turbulence is able to stabilise the thermally unstable gas making it able to last longer and consequently delaying the formation of the cold gas. In the same way, it is seen that the fraction of cold gas when statistical equilibrium is reached $(t=17.9$ and $20 \mathrm{Myr}$ ) is about $10 \%$ and thus significantly lower than for the cases $\epsilon=0.5$ and 2 . On the contrary, the fraction of thermally unstable gas is higher (about $30 \%$ ) and the drop at intermediate density $\left(\simeq 10 \mathrm{~cm}^{-3}\right)$ is less clear.

\subsection{Analysis of the CNM structures}

\subsubsection{The analysis}

We now investigate the properties of the CNM structures in more detail. We compute their surface and aspect ratio, their internal and relative (to each other) velocity dispersion, as well as internal density, temperature and pressure distributions. We define a structure as a connected set of pixels having a density above a given threshold, $n_{\mathrm{s}}=30 \mathrm{~cm}^{-3}$. This definition has a clear physical meaning since the front that connects the 2 phases is stiff and therefore the structures do not depend very much on the arbitrary threshold $n_{\mathrm{s}}$.

Once having identified the structures, we compute the inertia matrix $I$ defined by $I_{x x}=\int \rho x^{2} \mathrm{~d} x \mathrm{~d} y, I_{y y}=\int \rho y^{2} \mathrm{~d} x \mathrm{~d} y$ and $I_{x y}=I_{y x}=\int \rho x y \mathrm{~d} x \mathrm{~d} y$. It admits the 2 eigenvalues $\lambda_{1} \geq \lambda_{2}$. The aspect ratio $r$ is then defined by $r=\sqrt{\lambda_{2} / \lambda_{1}}$. We also consider the velocity of the structure, $\boldsymbol{V}_{\text {struct }}$, its surface, $S$, average density, $\langle\rho\rangle$, temperature, $\langle T\rangle$, and internal dispersion velocity defined as:

$\delta V^{2}=\frac{1}{N} \sum_{i=1, N} \frac{\rho_{i}}{\langle\rho\rangle}\left(\boldsymbol{V}_{\text {struct }}-\boldsymbol{v}_{i}\right)^{2}$,

where $N$ is the number of pixels in the structure.

Last, we compute the temperature, density and pressure variance for a structure defined as:

$\delta T=\sqrt{\frac{1}{N} \sum_{i=1, N} \frac{\rho_{i}}{\langle\rho\rangle}\left(1-\frac{T_{i}}{\langle T\rangle}\right)^{2}}$.

\subsubsection{Morphology and properties of the CNM}

Figure 9 displays the distribution of the density, temperature, pressure and velocity of the structures as well as their variance (see Sect. 3.4.1, for the definitions). It also shows their surfaces 

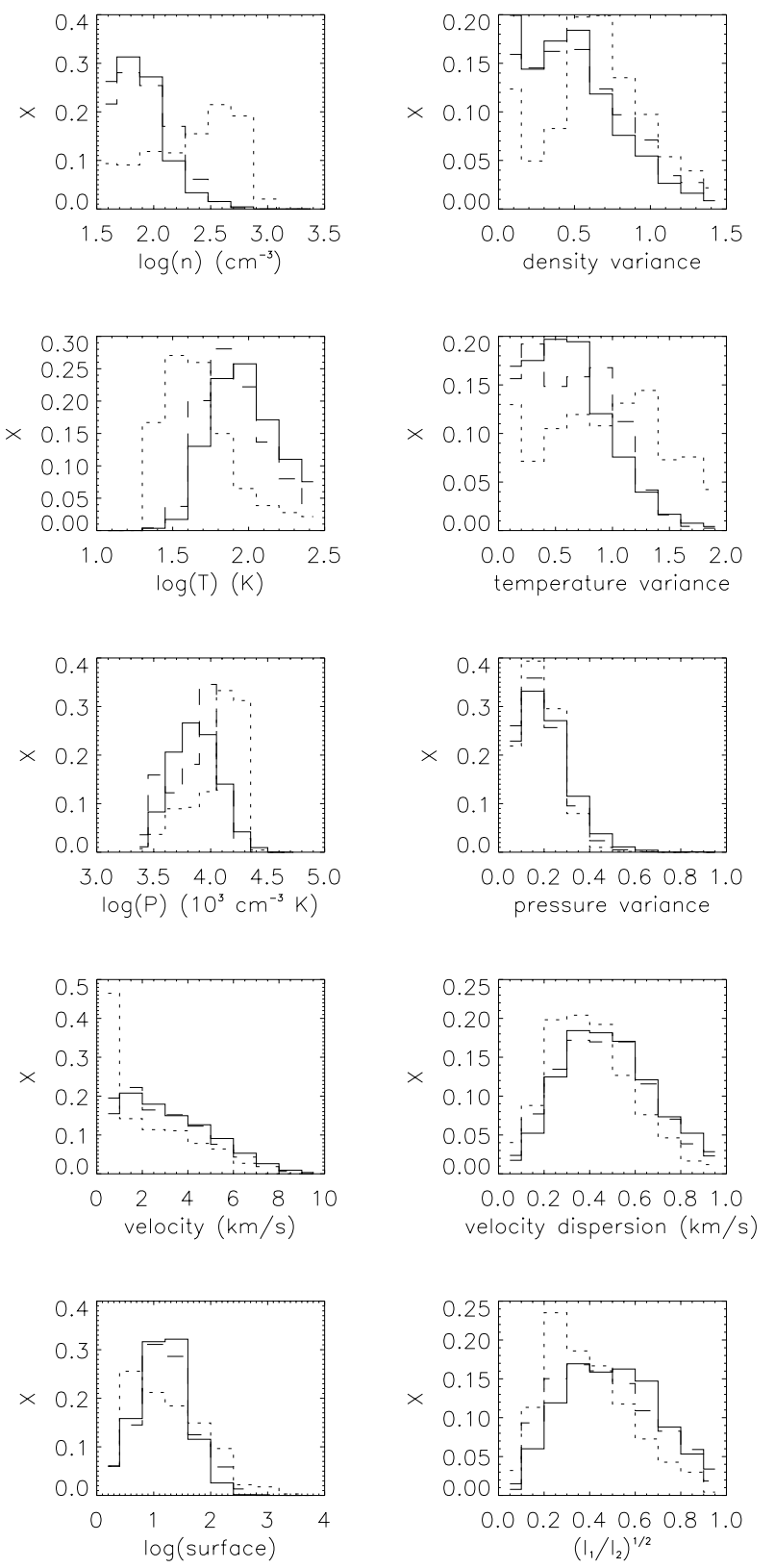

Fig. 9. Properties of CNM structures for the 3 cases $\epsilon=0.5$ (dashed line), $\epsilon=2$ (dot-dashed line) and $\epsilon=4$ (full line). The histograms display the density, temperature, pressure and velocity distributions as well as their variance, the size (in pixels) and the aspect ratio distribution. See text for definitions of these quantities.

(in pixels) and their aspect ratio. In order to obtain the distributions, we have added the structures obtained in 20 different time steps (separated by about $0.5 \mathrm{Myr}$ in time) leading to a total number of about 800 to 1500 structures of CNM (depending on the value of $\epsilon$ ).

The density histograms confirm the trend mentioned in the previous section that the structures are much denser in the weakly turbulent case than in the turbulent one by approximately a factor of 10 . They are consequently colder (by a factor of 3 ) and have a higher thermal pressure (by a factor of 3 ) for $\epsilon=0.5$ than for $\epsilon=4$.
The density and temperature variances (respectively $\simeq 0.6$ and $\simeq 0.7$ for $\epsilon=4$ and $\simeq 0.7$ and $\simeq 1$ for $\epsilon=0.5$ ) indicate that these quantities vary significantly inside one structure around their mean value. The pressure variance is significantly lower $(\simeq 0.3)$ which indicates that the structures are on average not far from mechanical equilibrium.

The structure velocity ranges from 0 to $8 \mathrm{~km} \mathrm{~s}^{-1}$ and is slightly lower in the weakly turbulent case $(\epsilon=0.5)$ than in the turbulent one. This is consistent with the results obtained by Koyoma \& Inutsuka (2002) who found a comparable velocity dispersion for their clouds. The internal velocity dispersion is also slightly lower in the weakly turbulent case, for which it peaks at $\simeq 0.35 \mathrm{~km} \mathrm{~s}^{-1}$, whereas it peaks at $\simeq 0.45 \mathrm{~km} \mathrm{~s}^{-1}$ for $\epsilon=4$. This indicates that most of the internal motions are subsonic with respect to the internal sound speed $\left(\simeq 0.8 \mathrm{~km} \mathrm{~s}^{-1}\right)$ of the CNM structures and is consistent with the small pressure variance.

Finally, it is seen that the mean surface of the structure is about 20-30 pixels which gives a typical length of about $\simeq 5$ pixels or $\simeq 0.1 \mathrm{pc}$. As already mentioned the structures are smaller when turbulence is higher. The structures have a mean aspect ratio of about 0.5 for $\epsilon=4$ and 0.35 for $\epsilon=0.5$.

\section{Turbulence and thermally unstable gas}

In this section we present a more detailed analysis of the role of turbulence in producing the filaments of thermally unstable gas discussed in the previous section. We first present a semianalytical model that describes the transition of a gas clump from the warm to the cold phase and propose an explanation for the origin of the unstable gas. We then compare the predictions of the model with the numerical results.

\subsection{A semi-analytical model for the evolution of a fluid particle}

\subsubsection{Formalism and physical interpretations}

In order to understand how turbulence generates thermally unstable gas, we have developed a semi-analytical model which is presented in Appendix A. The underlying physical idea of the model is simply to follow a fluid element and to calculate the evolution of its density and temperature by computing the cooling and heating of the gas and its geometrical evolution. It is based on a Lagrangian form of Eqs. (1)-(3) and the assumptions that the fluid element can be simply described by its semi-minor and major axis length $a(t)$ and $b(t)$, its internal pressure $P(t)$, the external pressure $P_{\text {ext }}$ and its density $\rho(t)$. With these assumptions the fluid element can be described by the following equations:

$$
\begin{aligned}
& \dot{\rho}+\rho \theta=0, \\
& \dot{\mathcal{E}}+P / \rho \theta=-\frac{1}{\rho} \mathcal{L}(\rho, T), \\
& \dot{\theta}+\theta^{2} / 2+2 \Sigma^{2} \simeq-\frac{P_{\mathrm{ext}}-P(t)}{\rho(t)}\left(\frac{1}{a(t)^{2}}+\frac{1}{b(t)^{2}}\right), \\
& \dot{\Sigma}+\theta \Sigma \simeq-\frac{1}{2} \frac{P_{\mathrm{ext}}-P(t)}{\rho(t)}\left(\frac{1}{a(t)^{2}}-\frac{1}{b(t)^{2}}\right) .
\end{aligned}
$$


In these equations $\theta$ is the divergence of the velocity field, $\Sigma=0.5\left(\partial_{x} u_{x}-\partial_{y} u_{y}\right)$ (where the axis $x$ and $y$ are the eigenframe of the shear tensor, see Appendix $), a(t)=a_{0} \exp \left(\int_{0}^{t}(\theta / 2+\Sigma) \mathrm{d} t\right)$ and $b(t)=a_{0} \exp \left(\int_{0}^{t}(\theta / 2-\Sigma) \mathrm{d} t\right)$. Note that $\Sigma$ describes the straining motions (e.g. Acheson 1992) which are divergence free and involve stretching and squashing in mutually perpendicular directions.

There are three characteristic time scales that can be associated with a collapsing fluid element. The cooling time, $\tau_{\text {cool }}$ (see Appendix), and two dynamical time scales: $\tau_{\text {dyn }}^{ \pm}=-\theta / 2 \pm \Sigma$ which correspond to the collapse time along the two principal axes of the fluid element. In the case of an isobaric evolution (see Appendix, for details), we have $\tau_{\text {cool }} \simeq \tau_{\text {dyn }}$ and the characteristic time of evolution of the fluid element will be the cooling time. If one takes the pressure gradient into account, the different time scales are not necessarily equal and more complex situations can arise.

When there is no turbulence the kinetic energy of the incoming flow is mainly converted into thermal pressure at the shock while in the turbulent case it is converted both into thermal pressure and turbulent motions. Consequently, the shocked gas in the low turbulence simulation has a very short cooling time (because of its high pressure) and a very long dynamical time because most of the kinetic energy was turned into thermal pressure. On the contrary, in the highly turbulent simulation, the shocked gas has a much longer cooling time and a smaller dynamical time.

In particular, if a dynamical time scale is shorter than the cooling time, it means that an axis is collapsing faster than the gas can cool. Therefore the pressure and the pressure gradient will rise along this particular direction which will eventually bounce and start to expand.

In view of this, it is possible to identify two physical mechanisms that explain why thermally unstable gas is generated by turbulence.

First, as illustrated by Figs. 3 and 7, the thermal pressure is higher in the case of a weakly turbulent flow than in the case of a very turbulent flow making the cooling time much shorter in the first case than in the second.

The second mechanism, which enhances the fraction of thermally unstable gas, is a dynamical one related to the straining motions. Let us consider for reference an initially spheroidal piece of thermally unstable gas which contracts isotropically. In such case $\theta$ is negative and $\Sigma$ vanishes. The density, $\rho$, increases and because of the cooling, the internal energy decreases. Therefore if the dynamical time scale is larger than the cooling time, the pressure $P$ decreases as well and the gas keeps contracting. In this case the dynamical times are equal on both axis. Let us now consider the same piece of gas but with a positive $\Sigma$. The dynamical time scale will be reduced on the $y$-axis and enhanced on the $x$-axis. The cooling time will remain the same (at least in the first phase) since the evolution of the density and of the pressure depend only on $\theta$. However, since the rate of contraction along the $y$-axis is higher, the pressure gradient will also be greater and the contraction can be slowed or even turned into expansion.

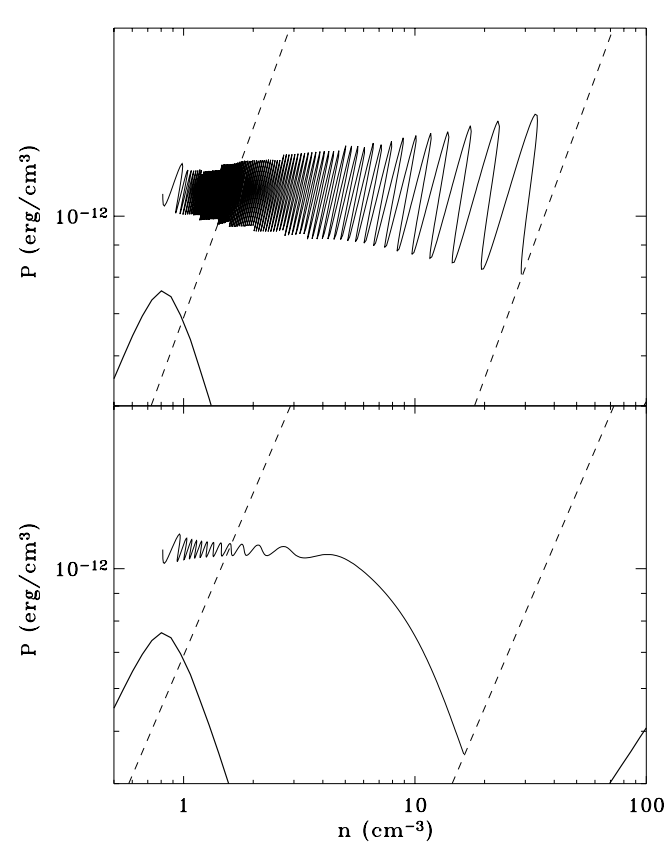

Fig. 10. Evolution of the semi-analytical model for a model with $(\theta, \Sigma)=(0,0)$ (bottom panel) and for the same model with $(\theta, \Sigma)=$ $\left(0,0.8 / \tau_{\text {cool }}\right)$ (upper panel). The dashed lines are the $5000 \mathrm{~K}$ and $200 \mathrm{~K}$ isothermal curves.

These dynamical effect are amplified by the thermally unstable nature of the gas. If for any dynamical reason the contraction is reduced, then the density will be lower and since $\partial_{\rho} P<0$ the pressure will be higher and it will therefore be even more difficult to collapse.

This stabilisation of unstable gas is illustrated in Fig. 10 where the evolution of a fluid element in the pressure-density diagram is displayed. The bottom panel is for $(\theta, \Sigma)=(0,0)$, whereas the upper panel is for $(\theta, \Sigma)=\left(0,0.8 / \tau_{\text {cool }}\right)$. Since we want to illustrate the dynamical effect of $\Sigma$, we have kept the initial thermal pressure identical for the two models but as was mentioned earlier, in the simulation the initial thermal pressure is lower on average when $\Sigma$ is large. It is seen that in the first case, the gas contracts rapidly with almost no oscillation and the thermal pressure decreases rapidly. In the second case the fluid element oscillates rapidly and the evolution is more isobaric. The fluid particle remains in the unstable domain about twice the time it spends in the unstable domain when $\Sigma=0$.

Finally, we stress that both mechanisms presented to explain the presence of unstable gas are related to the presence of turbulence. It is expected that filamentary structures will be produced by such motions. Since straining motions (i.e. $\Sigma$ ) are produced in a turbulent flow, this mechanism explains how turbulence may produce filaments of thermally unstable gas.

\subsubsection{Predictions of the semi-analytical model}

We now present the quantitative predictions of Eqs. (7)-(10) for the time spent in the thermally unstable domain. We consider a fluid element of warm gas after it has been shocked and we follow its evolution until it reaches a temperature of $200 \mathrm{~K}$. 


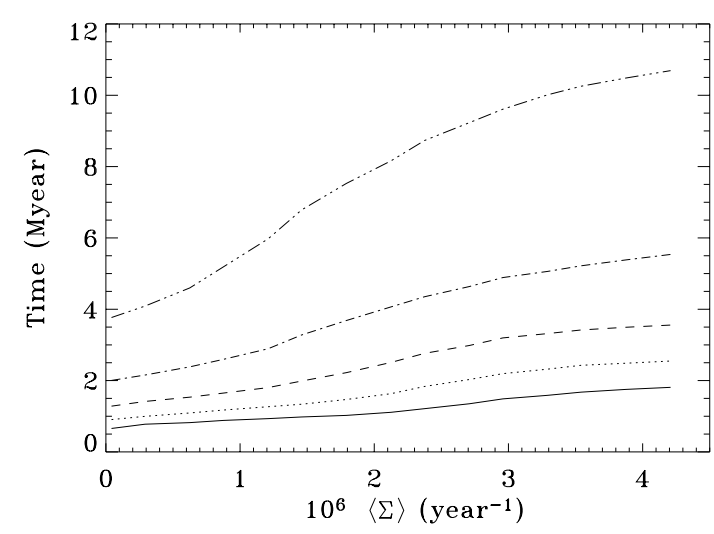

Fig. 11. Time spent by the fluid particle in the thermally unstable domain (defined as $200 \mathrm{~K}<T<5000 \mathrm{~K}$ ) during its contraction as a function of $\Sigma$. The 5 curves represent (from top to bottom) a Mach number of 1.2, 1.4, 1.6, 1.8 and 2.

We explore a large ensemble of initial conditions defined by the initial state of the gas (temperature and density), the initial values of $\theta$ and $\Sigma$ as well as the size of the fluid particle, $l$.

Figure 11 shows the mean time spent in the thermally unstable domain as a function of the initial value of $\Sigma$ for 5 values of the initial mach number, $M$, namely $1.2,1.4,1.6,1.8$ and 2 . The initial density and temperature are obtained by applying the Rankine-Hugoniot relations for an isothermal shock of mach number $M$ and preshocked gas of density and temperature equal to the values used as initial conditions in our simulations i.e. $n_{0}=0.76 \mathrm{~cm}^{-3}$ and $T_{0}=7100 \mathrm{~K}$. For each value of $M$ and $\Sigma$, we calculate the particle evolution for $\theta$ ranging from -5.0 to $2.5 \mathrm{Myr}^{-1}$, for $l$ ranging from 0.5 to $10 \mathrm{pc}$ and compute the mean value.

It is seen that, as expected, the time spent by the fluid particle in the thermally unstable domain, $\tau_{\mathrm{u}}$, decreases when the Mach number increases and increases when $\Sigma$ increases. The ratio between these times for $M=2$ and $\Sigma=0$ and $M=1.2$ and $\Sigma=4$ is about 10 .

Under simple assumptions, the results presented in Fig. 11 can be used to predict the relative ratio between the fraction of unstable and cold gas for different values of $M$ and $\Sigma$. Let $m_{\mathrm{u}}$ and $m_{\mathrm{c}}$ be the mass of the unstable and cold gas respectively. The cold gas forms from the collapse of the unstable gas and disappears when it reaches the upper or lower sides of our computational box. Therefore one has:

$\frac{\mathrm{d} m_{\mathrm{c}}}{\mathrm{d} t} \simeq \frac{m_{\mathrm{u}}}{\tau_{\mathrm{u}}}-\frac{m_{\mathrm{c}}}{\tau_{\mathrm{c}}}$

where $\tau_{\mathrm{c}}$ is the mean time spent by the cold gas in our simulation box. Therefore when statistical equilibrium is reached in our box, we have:

$\frac{\tau_{\mathrm{u}}\left(M_{1}, \Sigma_{1}\right)}{\tau_{\mathrm{u}}\left(M_{2}, \Sigma_{2}\right)} \simeq\left(\frac{m_{\mathrm{u}}}{m_{\mathrm{c}}}\right)_{\left(M_{1}, \Sigma_{1}\right)} /\left(\frac{m_{\mathrm{u}}}{m_{\mathrm{c}}}\right)_{\left(M_{2}, \Sigma_{2}\right)}$,

where we have assumed that $\tau_{\mathrm{c}}$ does not vary significantly with $M$ and $\Sigma$.

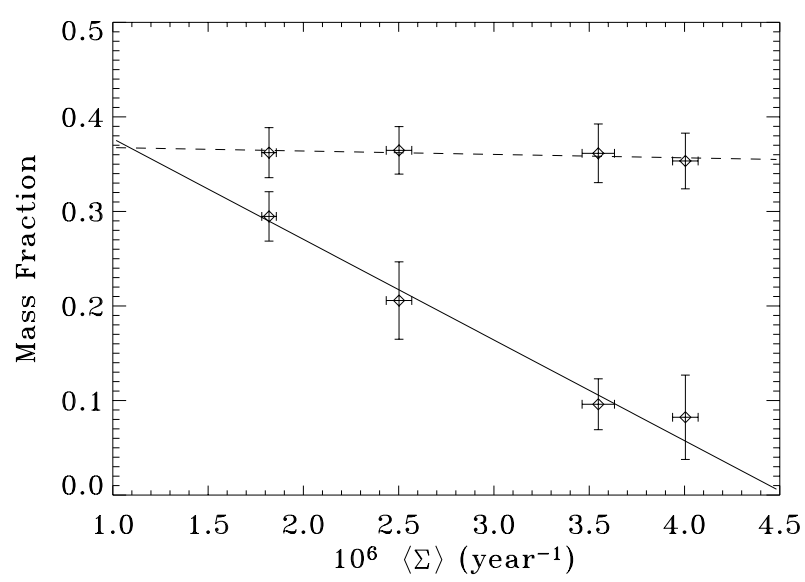

Fig. 12. Fraction of cold gas (full line) and fraction of thermally unstable gas plus cold gas (dashed line) as a function of $\langle\Sigma\rangle$ for the 4 cases $\epsilon=0.5,2,4$ and 6 for various time steps. Each point corresponds to the average over several outputs of a simulation with a given $\epsilon$ and the error bars show the total dispersion of the results $(100 \%$ of the points are within the error bar).

\subsection{Comparison with the numerical results}

In the model presented previously turbulence is able to transiently stabilise a piece of thermally unstable gas making it able to survive longer. This is done through the mechanisms presented in the previous section, which are both related to the presence of straining motions (i.e. $\Sigma$ ). Therefore this model predicts that the thermally unstable gas should be correlated with the parameter $\Sigma$ in the numerical simulations. In order to verify this effect we have smoothed the velocity field at a scale of $0.2 \mathrm{pc}$ (10 pixels) and computed $\Sigma$ at each pixel (note that larger smoothing scales, e.g. 0.4 or 1 pc, lead to similar results). Then the correlation between $\Sigma$ and the fluid temperature was investigated both globally and locally.

Figure 12 displays the total fraction of cold gas (full line) and the total fraction of cold plus thermally unstable gas (dashed line) in the 4 simulations $\epsilon=0.5,2,4$ and 6 at different times as a function of $\langle\Sigma\rangle$. It is seen that the second one is almost independent of $\langle\Sigma\rangle$ whereas the first decreases linearly with it. This result suggests that on one hand, the fraction of warm gas which is driven into the unstable regime depends mainly on the strength of the converging flow and is roughly independent of the level of turbulence. On the other hand, the thermally unstable gas is able to live longer when $\Sigma$ is stronger. For $\epsilon=0.5$, one has $\langle\Sigma\rangle \simeq 1.5 \times 10^{-6}$ year $^{-1}, m_{\mathrm{u}} / m_{\mathrm{c}} \simeq 0.32$ whereas the mean pressure of the warm gas before it starts contracting in $\mathrm{CNM}$ is about $3 \times 10^{-12} \mathrm{erg} / \mathrm{cm}^{3}$ (obtained from Fig. 3) corresponding to a Mach number $M$, of $\simeq 1.8$. For $\epsilon=4,\langle\Sigma\rangle \simeq 3.5 \times 10^{-6}, M \simeq 1.2$ (obtained from Fig. 7) and $m_{\mathrm{u}} / m_{\mathrm{c}} \simeq 2.8$. The relation between the pressure and the Mach number are obtained using isothermal Rankine-Hugoniot relations, which seems a reasonable assumption looking at Figs. 3 and 7 . The ratio between $m_{\mathrm{u}} / m_{\mathrm{c}}$ for these 2 values is therefore about 8 .

Let us make a quantitative comparison with the prediction of the semi-analytical model. Figure 11 predicts for the case of the simulation with $\epsilon=0.5(M \simeq 1.8$ and 

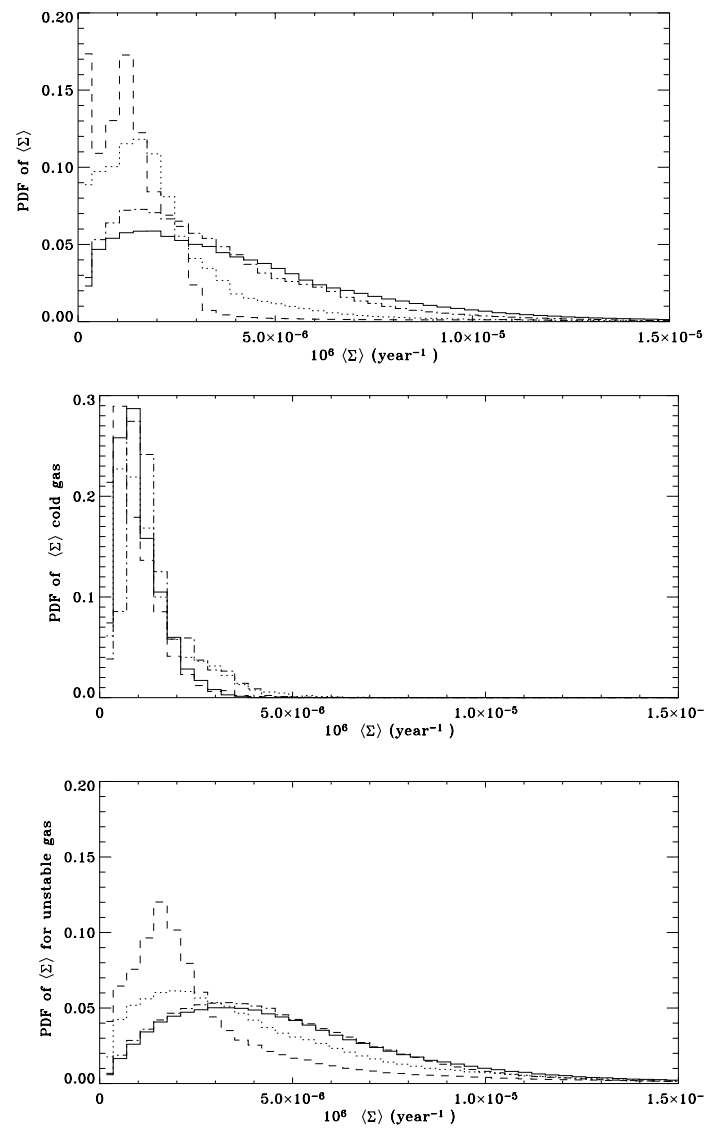

Fig. 13. Probability distribution function (pdf) of $\Sigma$ for $\epsilon=0.5$ (dashed line), $\epsilon=2$ (dotted line), $\epsilon=4$ (dashed-dotted line) and $\epsilon=6$ (full line). Upper panel is for the whole simulation, middle panel for cold gas only and lower panel for thermally unstable gas.

$\langle\Sigma\rangle \simeq 1.5 \times 10^{-6}$ year $\left.^{-1}\right), \tau_{\mathrm{u}} \simeq 1.5 \mathrm{Myr}$ whereas for $\epsilon=4$ $\left(M \simeq 1.2\right.$ and $\langle\Sigma\rangle \simeq 3.5 \times 10^{-6}$ year $\left.^{-1}\right)$ it predicts about $10 \mathrm{Myr}$. According to the arguments presented in Sect. 4.1.2, this leads to an estimate for the ratio between $m_{\mathrm{u}} / m_{\mathrm{c}}$ in these 2 cases of about $10 / 1.5 \simeq 7$ which is in reasonable agreement with the value $(\simeq 8)$ measured in the numerical experiment.

Figure 13 displays the probability distribution function (pdf) of $\Sigma$ for $\epsilon=0.5$ (dashed line), $\epsilon=2$ (dotted line), $\epsilon=4$ (dot-dashed line) and $\epsilon=6$ (full line). The upper panel is for the whole simulation whereas the middle one is restricted to cold gas $(T<200 \mathrm{~K})$ and the lower to thermally unstable gas $(200 \mathrm{~K}<T<5000 \mathrm{~K})$. It is obvious that in the cold phase, $\Sigma$ is much lower than for the thermally unstable gas which is associated with high values of $\Sigma$. Note that for $\epsilon=0.5$, the thermally unstable gas has a lower $\Sigma$ than for $\epsilon=4$. This is consistent with the fact that this is turbulence that generates flows having large values of $\Sigma$.

Both results are, qualitatively and quantitatively, in good agreement with the explanation proposed in the previous sections. Another important, although qualitative, fact is that the thermally unstable gas is organised in filaments which is also a natural outcome of a mechanism based on straining motions.

\section{Conclusion}

We perform 2-d numerical simulations to understand the dynamics of thermally bistable interstellar atomic hydrogen. The size of our $1000^{2}$ simulation box is $20 \mathrm{pc}$, leading to a numerical resolution of $0.02 \mathrm{pc}$. Such resolution is needed in order to properly describe the cold HI structures. In order to mimic a large-scale converging flow that triggers the transition of the warm phase into the cold one, warm gas is injected continuously from 2 opposite sides of the box. The gas can freely escape at the 2 other boundaries. Various amplitudes of turbulent fluctuations have been applied.

When the flow is weakly turbulent, a layer of compressed WNM forms and quickly fragments into structures of cold gas. When the flow is very turbulent, the geometry is more complex. A large fraction of thermally unstable gas which increases with the amplitude of the turbulent forcing, is found. This thermally unstable gas tends to be organised in filamentary structures. Nevertheless the thermally bistable behaviour is not erased and indeed at a scale comparable to the size of the CNM structures very similar to the classical picture of the 2-phase medium. In particular, the 2 phases are connected through sharp thermal fronts and are locally in rough pressure equilibrium. Of course the CNM structures undergo shocks and large fluctuations but even when it is the case, the 2 phases may still be clearly identified. We therefore propose that the atomic hydrogen may be accuralety defined as a dynamical 2-phase medium.

In order to characterise the CNM structures found in the numerical simulation, we apply a simple threshold algorithm to identify them and give the pdf of some of their intrinsic properties such as mean density, temperature, velocity dispersion, size and aspect ratio, $r$. Typical values for these parameters are respectively: $\langle n\rangle \simeq 50 \mathrm{~cm}^{-3}, T \simeq 80 \mathrm{~K}, \delta v \simeq 0.5 \mathrm{~km} \mathrm{~s}^{-1}$, $l \simeq 0.1 \mathrm{pc}$ and $r \simeq 0.5$.

A semi-analytical model for the thermal and dynamical evolution of a fluid particle is presented. It is based on the Lagrangian description of a fluid element. This model predicts that substantial straining motions may efficiently stabilise a piece of thermally unstable gas allowing it to survive a longer period of time. We then verify than both locally and globally the thermally unstable gas in the numerical simulations is indeed very strongly correlated with substantial straining motions.

Acknowledgements. We acknowledge the support of the CEA computing center, CCRT, where all the simulations where carried out. We also would like to thank Jean-Michel Alimi and Jean-Pierre Chièze for stimulating discussions. We acknowledge a critical reading of the manuscript by Michel Pérault and Enrique Vàzquez-Semadeni as well as enlighting discussions on the results presented in this manuscript. P.H. gratefully acknowledges the support of a CNES fellowship.

\section{References}

Acheson, D. J. 1992, Elementary Fluid Dynamics (Oxford Applied Mathematics and Computing Science Series)

Ballesteros-Paredes, J., Vázquez-Semadeni, E., \& Scalo J. 1999, ApJ, 515,286

Boulanger, F., \& Pérault, M. 1988, ApJ, 330, 964 
Dahlburg, R., DeVore, C., Picone, J., Mariska, J., \& Karpen, J. 1987, ApJ, 315, 385

Draine, B. 1978, ApJS, 36, 595

Field, G. 1965, ApJ, 142, 531

Field, G., Goldsmith, D., \& Habing, H. 1969, ApJ, 155, L149

Gazol, A., Vázquez-Semadeni, E., Sánchez-Salcedo, F., \& Scalo, J. 2001, ApJ, 557 L124

Heiles, C. 1997, ApJ, 481, 193

Hennebelle, P., \& Pérault, M. 1999, A\&A, 351, 309

Hennebelle, P., \& Pérault, M. 2000, A\&A, 359, 1124

Joncas, G., Boulanger, F., \& Dewdney, P. E. 1992, ApJ, 397, 165

Karpen, J. T., Picone, J. M., \& Dahlburg, R. B. 1988, ApJ, 324, 590

Korpi, M. J., Brandenburg, A., Shukurov, A., et al. 1999, ApJ, 514, L99

Kovalenko, I. G., \& Shchekinov, Y. A. 1999, Phys. Plasmas, 6, 335

Koyoma, H., \& Inutsuka, S. 2000, ApJ, 532, 980

Koyoma, H., \& Inutsuka, S. 2002, ApJ, 564, L97

Koyoma, H., \& Inutsuka, S. 2004, ApJ, 602, L25

Kritsuk, A. G., \& Norman, M. L. 2002a, ApJ, 569, L127

Kritsuk, A. G., \& Norman, M. L. 2002b, ApJ, 580, L51
Kulkarni, S. R., \& Heiles, C. 1987, Interstellar processes, ed. Hollenbach D., Thronson H. (Reidel)

Low, F. J., Beintena, D. A., Gautier, T. N., et al. 1984, ApJ, 278, L19 McKee, C. F., \& Ostriker, J. P. 1977, ApJ, 218, 448

Meerson, B. 1996, Rev. Mod. Phys., 68, 215

Parravano, A., Hollenbach, D. J., \& McKee, C. F. 2002, ApJ, 584, 797

Passot, T., Vázquez-Semadeni, E., \& Pouquet, A. 1995, ApJ, 441, 702

Penston, M., \& Brown, F. 1970, MNRAS, 150, 373

Piontek, R. A., \& Ostriker, E. C. 2004, ApJ, 601, 905

Sánchez-Salcedo, F. J., Vázquez-Semadeni, E., \& Gazol, A. 2002, ApJ, 577, 768

Troland, T., \& Heiles, C. 2003, ApJ, 586, 1067

Vázquez-Semadeni, E., Passot, T., \& Pouquet, A. 1996, ApJ, 473, 881

Vázquez-Semadeni, E., Gazol, A., Passot, T., \& Sánchez-Salcedo, J. 2003 in Turbulence and magnetic fields in astrophysics, ed. E. Falgarone and T. Passot (Springer)

Wolfire, M. G., Hollenbach, D., \& McKee, C. F. 1995, ApJ, 443, 152

Wolfire, M. G., Hollenbach, D., \& McKee, C. F. 2003, ApJ, 587, 278

Zel'dovich, Y., \& Pikel'ner, S. 1969, JETP, 29, 170 
E. Audit and P. Hennebelle: Thermal condensation in atomic hydrogen, Online Material p 1

\section{Online Material}




\section{Appendix A: A semi-analytical model}

In this appendix we derive the semi-analytical model that we use in Sect. 4. The idea of the model is to follow a fluid element by writing Eqs. (1)-(3) in a Lagrangian form and then making some approximations to compute the pressure gradients.

The conservation of the mass can be written in a Lagrangian form as:

$\dot{\rho}+\rho \theta=0$.

where $\theta$ is the divergence of the velocity field and the dot represents a total (i.e. Lagrangian) derivative. The evolution of thermal energy can also be simply written in a Lagrangian form:

$\dot{\mathcal{E}}+\frac{P}{\rho} \theta=\frac{1}{\rho} \mathcal{L}(\rho, T)$,

where $\mathcal{E}=P /(\rho(\gamma-1))$ is the specific thermal energy.

In order to have an evolution equation for $\theta$ we take the gradient of Eq. (2). As it is common in fluid mechanics, we then decompose the resulting tensorial equation into its trace, symmetric trace-free and anti-symmetric part. For the sake of simplicity and since we want to compare our model to the simulation presented above, we write these equations for a bidimensional flow:

$$
\begin{aligned}
& \dot{\theta}+\frac{\theta^{2}}{2}+2\left(\sigma_{1}^{2}+\sigma_{2}^{2}-\omega^{2}\right)=-\left(P_{x x}+P_{y y}\right), \\
& \dot{\sigma_{1}}+\theta \sigma_{1}=-\frac{1}{2}\left(P_{x x}-P_{y y}\right), \\
& \dot{\sigma_{2}}+\theta \sigma_{2}=-\frac{1}{2}\left(P_{x y}+P_{y x}\right), \\
& \dot{\omega}+\theta \omega+\omega\left(\sigma_{2}-\sigma_{1}\right)=0,
\end{aligned}
$$

where $\sigma_{1}=0.5\left(\partial_{x} u_{x}-\partial_{y} u_{y}\right)$ and $\sigma_{2}=0.5\left(\partial_{x} u_{y}+\partial_{y} u_{x}\right)$ are the two components of the shear tensor and $\omega=0.5\left(\partial_{x} u_{y}-\partial_{y} u_{x}\right)$ is the rotational. The $P_{i j}$ are defined by: $P_{i j}=\partial_{i}\left(\partial_{j} P / \rho\right)$.

The system (A.1)-(A.6) is exact but is not closed since there is no evolution equation for $P_{i j}$. Therefore it cannot be integrated unless one makes some approximation to compute the $P_{i j}$.
The simplest case to consider, as a starting point, is the evolution of an isobaric fluctuation. In that case $P_{i j}=0$ and the evolution of the fluid element is given by:

$\theta=-\frac{\dot{\rho}}{\rho}=\frac{(\gamma-1)}{\gamma P_{0}} \mathcal{L}(\rho, T)=-\frac{\dot{\sigma_{1}}}{\sigma_{1}}=-\frac{\dot{\sigma_{2}}}{\sigma_{2}}$,

where $P_{0}$ is the constant pressure and $e_{0}=P_{0} /(\gamma-1)$ is the corresponding internal energy. The cooling time, $\tau_{\text {cool }}=$ $-e_{0} / \mathcal{L}(\rho, T)$ is the characteristic time of evolution of the system.

In order to treat the pressure gradient let us first simplify the previous system. If rotation is neglected, the shear tensor can be diagonalized and the eigenvector basis will not rotate during the evolution. In this eigenframe, the only component of the shear tensor is given by $\Sigma=\sqrt{\sigma_{1}^{2}+\sigma_{2}^{2}}$ and the fluid element can be viewed as an ellipsoid defined by its semi-major and minor axis of length $a$ and $b$. The equation of evolution for $\Sigma$ is identical to that of $\sigma_{1}$ Eq. (A.4) with the pressure gradient taken in the eigenframe. The evolution of the ellipsoid axis is given by:

$a(t)=a_{0} \exp \left(\int_{0}^{t}(\theta / 2+\Sigma) \mathrm{d} t\right) \equiv a_{0} a_{\theta} a_{\Sigma}$,
$b(t)=b_{0} \exp \left(\int_{0}^{t}(\theta / 2-\Sigma) \mathrm{d} t\right) \equiv b_{0} a_{\theta} / a_{\Sigma}$,

where we have defined $a_{\theta}=\exp \left(\int_{0}^{t} \theta / 2 \mathrm{~d} t\right)$ and $a_{\Sigma}=$ $\exp \left(\int_{0}^{t} \Sigma \mathrm{d} t\right)$.

If we further assume that the pressure and density inside and outside the ellipsoid are uniform then we may write:

$P_{x x} \simeq \frac{P_{\mathrm{ext}}-P(t)}{\rho(t) a(t)^{2}}, \quad P_{y y} \simeq \frac{P_{\mathrm{ext}}-P(t)}{\rho(t) b(t)^{2}}$ and $P_{x y} \simeq 0$.

Using this approximation for $P_{i j}$, it is now possible to integrate the system (A.1)-(A.6) to determine the evolution of the fluid element. 